\title{
OPTICAL INVESTIGATIONS OF AIGaN ON GaN EPITAXIAL FILMS
}

\author{
G. Steude ${ }^{1}$, T. Christmann ${ }^{1}$, B.K. Meyer ${ }^{1}$, A. Goeldner ${ }^{2}$, A. Hoffmann ${ }^{2}$, F. Bertram ${ }^{3}$, J. Christen ${ }^{3}$, \\ H.Amano ${ }^{4}$, and I. Akasaki ${ }^{4}$ \\ ${ }^{1}$ 1.Physikalisches Institut, Universitaet Giessen, 35952 Giessen, Germany, \\ ${ }^{2}$ Institut für Festkoerperphysik der TU Berlin, Hardenbergstr. 36, D-10623 Berlin, Germany, \\ ${ }^{3}$ Otto von Guericke Universitaet Magdeburg, PF 4120, D-39016 Magdeburg, Germany \\ ${ }^{4}$ Department of Electrical and Electronic Engineering, 1-501 Shiogamaguchi, Tempaku-ku, \\ Nagoya 468, Japan
}

Cite this article as: MRS Internet J. Nitride Semicond. Res. 4S1, G3.26(1999)

\begin{abstract}
We have investigated $\mathrm{Al}_{\mathrm{x}} \mathrm{Ga}_{1-\mathrm{x}} \mathrm{N} / \mathrm{GaN}$ heterostructures $(0<\mathrm{x}<0.22)$ grown by metal organic vapor phase epitaxy on sapphire with photoluminescence (PL), reflexion and cathodo-luminescence experiments. The energetic positions of the free A-exciton in $\mathrm{GaN}$ and $\mathrm{AlGaN}$ as a function of the alloy compositions are deduced from temperature dependent PL and from reflexion measurements. We obtain a small bowing parameter and no evidence for a Stokes shift between absorption and emission. The $\mathrm{Al}_{\mathrm{x}} \mathrm{Ga}_{1-\mathrm{x}} \mathrm{N}$ films induce additional compressive strain on the underlying $\mathrm{GaN}$ film. Compositional inhomogeneities are present, but the fluctuations are too small to be important for carrier localisation. The broadening of the luminescence line width in the alloy can be described by statistical disorder of a random alloy.
\end{abstract}

\section{INTRODUCTION}

A wide field of applications can be expected from opto-electronic devices based on the group-III nitrides, AlN, GaN and InN. Together with their ternary alloys they can cover the visible to ultraviolet spectral range. In order to optimize the device performance especially the properties of the ternary alloys have to be understood and controlled. Since the lattice mismatch between AlN and $\mathrm{GaN}$ is $2.4 \%$ and for $\mathrm{InN}$ on $\mathrm{GaN}$ it is $11 \%$, strain effects (i.e. pseudomorphic versus strain relaxed growth) play an important role. They can lead to compositional fluctuations and eventually to phase separations. Also the influence of piezo electric field effects on the luminescent properties cannot be neglected.

We have recently reported on the excitonic luminescence of $\mathrm{Al}_{\mathrm{x}} \mathrm{Ga}_{1-\mathrm{x}} \mathrm{N}$ on $\mathrm{GaN}$ heterostructures and derived the excitonic band gap dependence on the Al molar fraction $(x<0.22)$ at room temperature ${ }^{1}$. The deviation from a linear dependence was small and we obtained the non-linear contribution (bowing parameter) to be $0.6 \mathrm{eV}$. At low temperatures bound exciton emission dominates $^{2}$. The free A-exciton emission was only observable at elevated temperatures ( $\mathrm{T}>100 \mathrm{~K})$. We have extended these investigation towards reflexion, transmission and absorption spectroscopy using calorimetric detection (CRS, CTS, CAS) to conclude on the absence or presence of a Stokes shift between absorption and emission. Cathodoluminescence experiments were used to resolve eventual compositional inhomogeneties. 


\section{EXPERIMENTAL DETAILS}

The films were grown by MOVPE on sapphire (0001) substrates. In a first step a $2 \mu \mathrm{m}$ thick $\mathrm{GaN}$ film was grown on a $30 \mathrm{~nm}$ low temperature deposited AlN buffer. On this GaN layer $\mathrm{Al}_{\mathrm{x}} \mathrm{Ga}_{1-\mathrm{x}} \mathrm{N}$ films with thicknesses between 350 and $650 \mathrm{~nm}$ were grown. The AlN molar fraction varied between 0 and 0.22 . More details especially about the determination of the composition can be found in ref. ${ }^{3}$ Details of the set up for calorimetric detection of reflexion, transmission and absorption spectroscopy can be found in ref. ${ }^{4} 4 \mathrm{~K}$ cathodoluminescence (CL) measurements were performed in a fully computerized scanning electron microscope. The spatial resolution is better then $50 \mathrm{~nm}$. In the CL wavelength image (CLWI) mode the local emission wavelength is mapped at any point of a rectangular area which is scanned by the electron beam. For further details see ref. $^{5}$ The luminescence was excited with an Excimer laser at $248 \mathrm{~nm}$.

\section{RESULTS AND DISKUSSION}

In fig. 1 the photoluminescence spectra of $\mathrm{Al}_{\mathrm{x}} \mathrm{Ga}_{1-\mathrm{x}} \mathrm{N}$ epilayers with $\mathrm{Al}$ contents from 6 to $22 \%$ are shown. Apart from the shift of the peak position with increasing Al content one also observes a considerable increase in the linewidth, which is caused by alloy broadening (see below). In all cases the free A-exciton and neutral donor bound exciton of the underlying GaN layer could be detected (see inset in fig.1). One notes that the line positions shift blue as a function of the Al composition which means that additional biaxial compressive strain is induced in the GaN layers. In the $\mathrm{Al}_{\mathrm{x}} \mathrm{Ga}_{1-\mathrm{x}} \mathrm{N}$ layers at temperatures around $100 \mathrm{~K}$ the free A-exciton emission appeared which then was observed up to $500 \mathrm{~K}$, the highest temperature wich could be achieved in our cryosystem. The temperature dependence of the excitonic gap was fitted by the following formula ${ }^{6,7}$ :

$\mathrm{E}(\mathrm{T})=\mathrm{E}(0)-\kappa /\left\{\exp \left(\Theta_{\mathrm{E}} / \mathrm{T}\right)-1\right\}$

with $\kappa=0.38$ and $\Theta_{\mathrm{E}}=618 \mathrm{~K} \pm 30 \mathrm{~K}$. The parameters were the same for all five samples (as one example see fig.2). In PL at low temperatures bound exciton emission and not free exciton

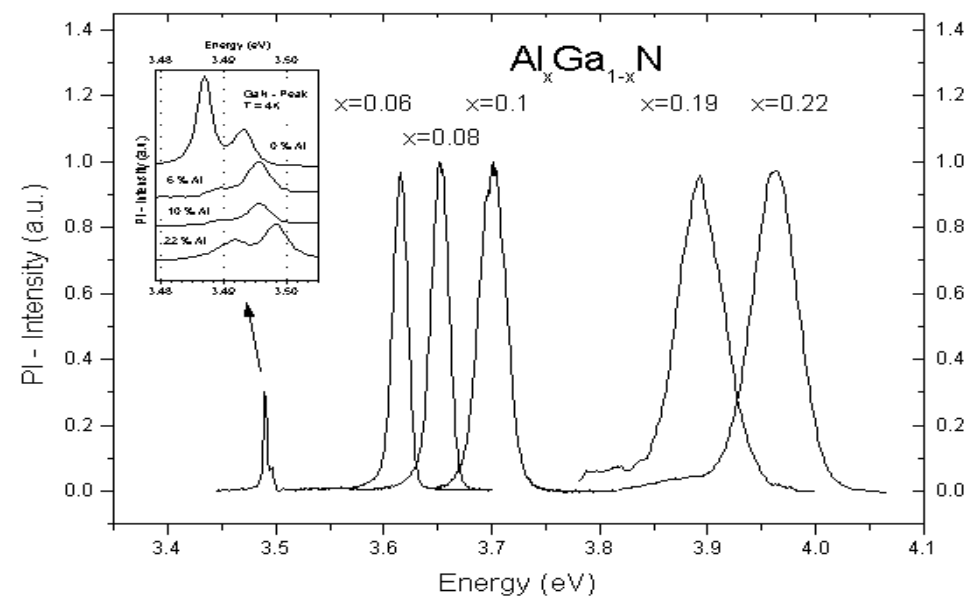

Fig.1: Photoluminescence of $\mathrm{Al}_{\mathrm{x}} \mathrm{Ga}_{1-\mathrm{x}} \mathrm{N}$ epilayers and of the underneath $\mathrm{GaN}$ films (inset). 


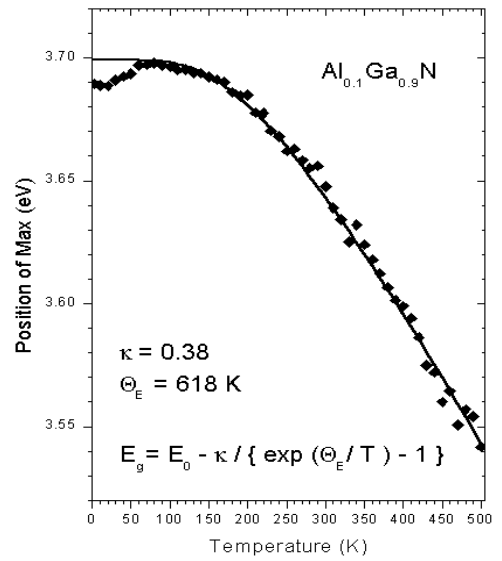

Fig.2: Measured band gap of $\mathrm{Al}_{0.1} \mathrm{Ga}_{0.9} \mathrm{~N}$ vs temperature. The drawn line is a fit to the experimental data (see text). emission dominates. One notes that the data points deviate at temperatures below $50 \mathrm{~K}$ from the fitted line. The excitons are now localized at neutral donors and there is an energetic difference between free and bound exciton emission, the localization energy. In order to obtain the free exciton line position at low temperatures we extrapolated from the high temperature behaviour to the low temperature behaviour using equ. 1 .

In fig. 3 we present a CAS spectrum of a sample with $6 \% \mathrm{Al}$ content. The measurement temperature was 47 $\mathrm{mK}$. One can identify the signals from the A- and Bexcitons of the underlying $\mathrm{GaN}$ at the band gap energy of around $3.5 \mathrm{eV}$. At higher energies the signal from the A-exciton of the AlGaN film can be seen. In fig.3 (inset) we compare the line positions of the free A-exciton from calorimetric measurements and PL. One notes an almost perfect agreement. Small differences might be caused by compositional inhomogeneities which we tried to resolve by CL measurements.

In fig. 4 we present CLWI of the film with an $\mathrm{Al}$ content of $\mathrm{x}=0.06$. On a scale of $10 \mathrm{x} 20 \mu^{2}$ the spectral position of the luminescence is mapped. In the wavelength image spectra are measured spot by spot and the peak positions found are translated into a colour scale. Blue means emission at $341.5 \mathrm{~nm}$ and yellow at $342.5 \mathrm{~nm}$. One can certainly see inhomogeneities on the scale of micrometers (fig.4.b). One should, however, have in mind that the energetic positions differ only by some 5 to $10 \mathrm{meV}$. In the integrated intensity picture (fig.4.c) areas of bright emission (yellow) and low emission efficiency (blue) can be distinguished. The blue areas are caused by structural imperfections on the surface such as scratches. They can also be identified in the scanning electron microscope (SE) image (fig.4.a). If all spectra are added up, the averaged spectrum results (fig.4.d). The CL results were very similar for all five $\mathrm{Al}_{\mathrm{x}} \mathrm{Ga}_{1-\mathrm{x}} \mathrm{N}$ films, and it is worth to note that the film with $\mathrm{x}=0.22$ had very much the same scatter in line positions (10 to 15

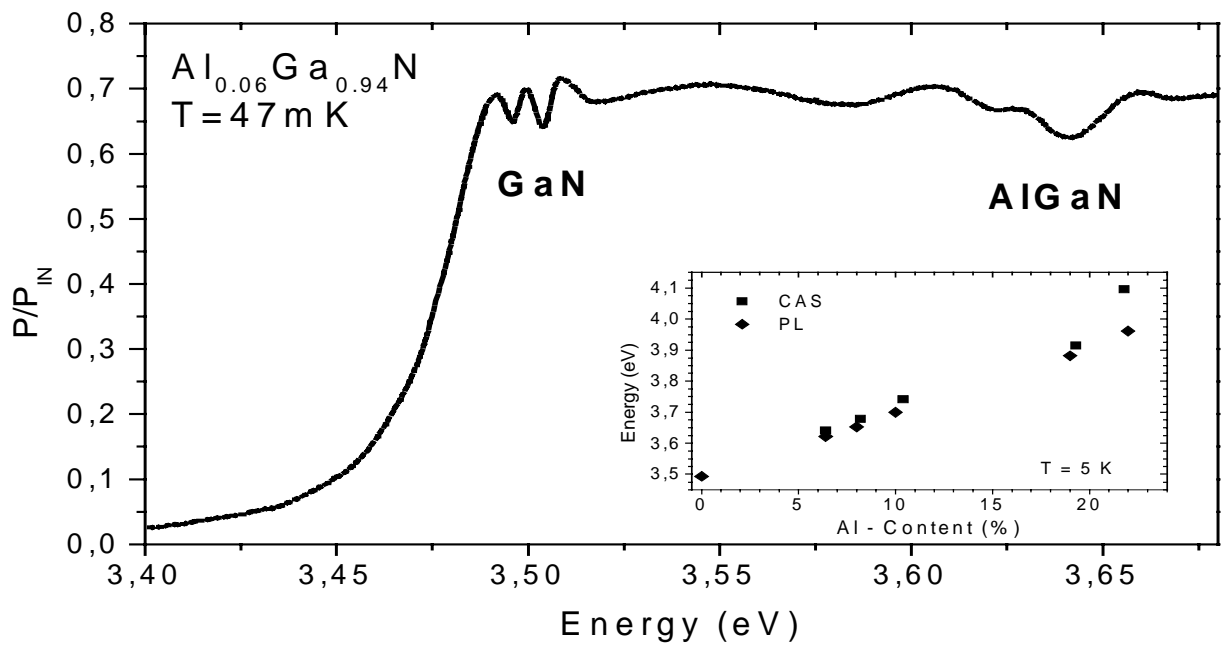

Fig.3: Calorimetric absorption spectrum of a $\mathrm{Al}_{0.06} \mathrm{Ga}_{0.94} \mathrm{~N}$ epilayer. In the inset the free Aexciton line position as a function of $\mathrm{Al}$ composition is shown. 

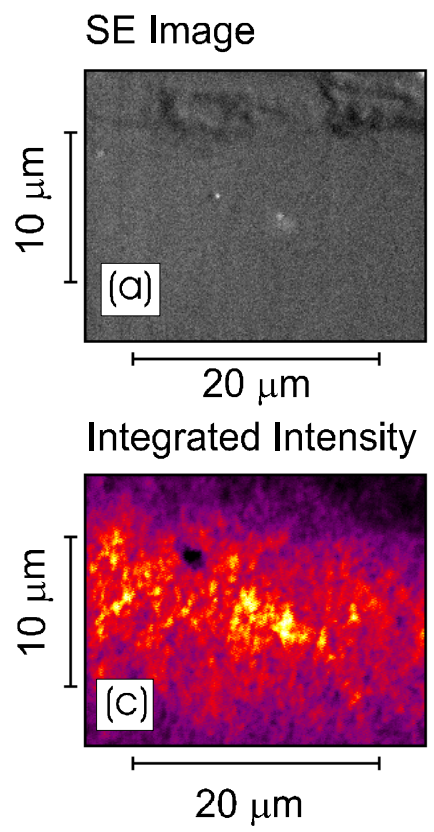

Fig.4: Catodoluminescence results on a $\mathrm{Al}_{0.06} \mathrm{Ga}_{0.94} \mathrm{~N}$ epilayer (a) secondary electron image; (b) wavelength image; (c) integrated intensity; (d) averaged spectrum.

meV) as the film with $\mathrm{x}=0.06$.

This demonstrates that in $\mathrm{Al}_{\mathrm{x}} \mathrm{Ga}_{1-\mathrm{x}} \mathrm{N} / \mathrm{GaN} \quad$ single heterostructures compositional inhomogeneities are less pronounced compared to InGaN/GaN.

Well resolved spectra of the A- and B-excitons in the underneath GaN films could be observed independent of the $\mathrm{Al}$ content by calorimetric spectroscopy. Their energetic positions as a function of the $\mathrm{Al}$ contents are shown in fig.5. There is a clear blue shift for both excitons, the energetic distance from $\mathrm{A}$ to $\mathrm{B}$ increases from $9 \mathrm{meV}$ for $\mathrm{x}=0.06$ to $11 \mathrm{meV}$ for $\mathrm{x}=0.22$. In the photoluminescence experiments only the A-exciton transition could be determined, the data are in agreement with the calorimetric measurements. From refexion and luminescence one can conclude that the top $\mathrm{Al}_{\mathrm{x}} \mathrm{Ga}_{1-\mathrm{x}} \mathrm{N}$ layer adds additional compressive strain to the underneath GaN film.

Strain modifies via the deformation potentials the eigen frequency of the phonons which can be measured by Raman spectroscopy. From the shift of the Raman lines in biaxially compressively strained films compared to the unstrained value the amount of built-in elastic strain can be quantified. Since the polar modes of E1 and A1 symmetry have a directional dispersion, the LO phonons interact with the vibrations of free carriers, and since the E2(low) has only a very small pressure coefficient, the E2(high) is the only usable mode for determination of the strain. Its shift is given by:

$\Delta \omega_{\mathrm{E} 2(\mathrm{high})}=4.2 \mathrm{~cm}^{-1} / \mathrm{GPa} \cdot \sigma_{\mathrm{xx}}$ with $\sigma_{\mathrm{xx}}$ the respective component of the strain tensor. We used the room temperature intrinsic value of $\omega_{\mathrm{E} 2(\mathrm{high})}=567 \mathrm{~cm}^{-1}$, which was determined for freestanding GaN films. There is a shift of the Raman line from the intrinsic value as as function of Al molar fraction. It is around 0.8 wavenumbers for $\mathrm{x}=0.06$ and around 1.4 wavenumbers for $\mathrm{x}=0.218$. These experimental findings could be quantified by using a two layer model (neglecting thermal mismatch) and taking into account the elastic constants of $\mathrm{Al}_{\mathrm{x}} \mathrm{Ga}_{1-\mathrm{x}} \mathrm{N}$ (interpolating between GaN and AIN). Details will be published elsewhere ${ }^{8}$.

Besides the energetic shift of the excitonic emission with increasing Al-content the linewidth of the bound exciton luminescence line increases from $14 \mathrm{meV}$ for $\mathrm{x}=0.06$ to $35 \mathrm{meV}$ for $\mathrm{x}=0.22$. This can be explained by alloy brodening. We compare the experimental values with a calculation based on a model introduced by Schubert et al. ${ }^{9}$ The broadening is caused by a statistical occupation of the cation places $\mathrm{Ga}$ by $\mathrm{Al}$ and the alloy disorder is seen by the exciton. Therefore we need to consider the exciton volume and its Bohr radius. The alloy broadening is given by: 


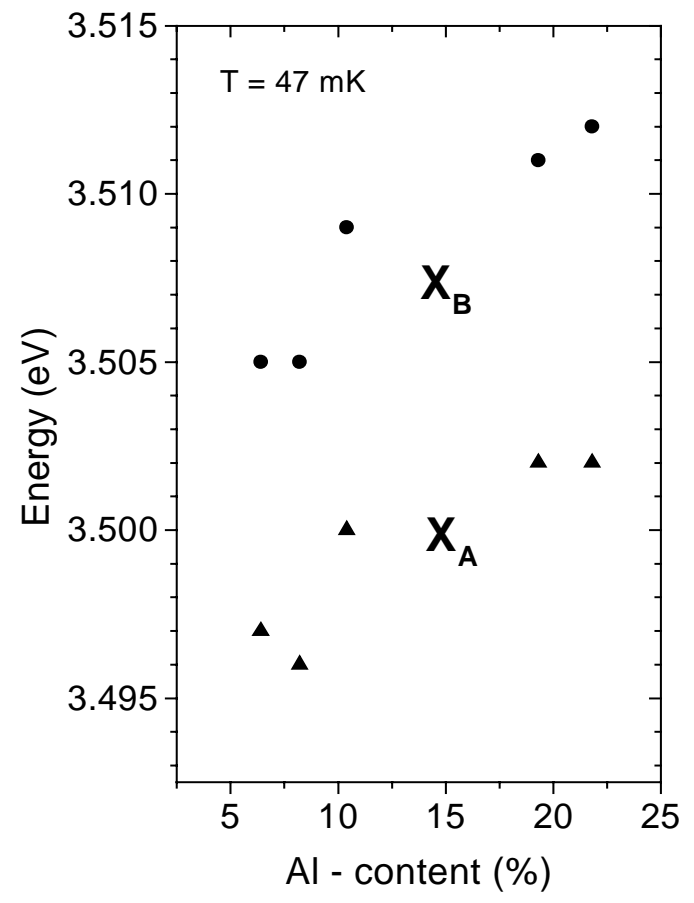

Fig.5: Line positions of the free A- and Bexcitons in $\mathrm{GaN}$ as a function of $\mathrm{Al}$ content in $\mathrm{Al}_{\mathrm{x}} \mathrm{Ga}_{1-\mathrm{x}} \mathrm{N}$ on $\mathrm{GaN}$ epitaxial films.

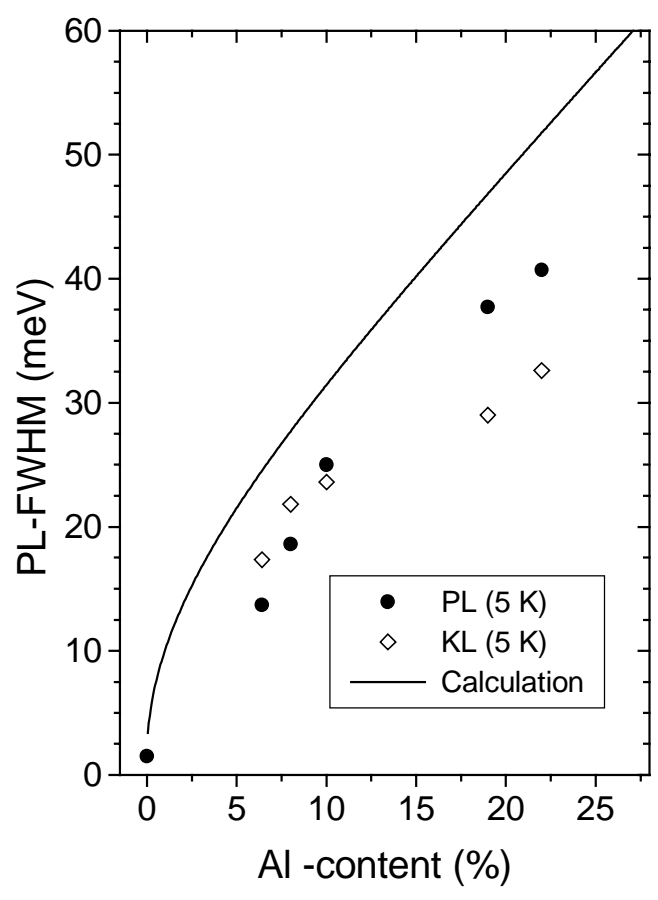

Fig.6: Alloy broadening of the photoluminescence line width (PL: full circles; CL: hollow diamonds). The drawn line shows a calculation using equ.(2).

$\Delta \mathrm{E}_{\mathrm{exc}}=2.36 \sigma_{\mathrm{E}}=2.36 \mathrm{dE} / \mathrm{dx}\left\{\mathrm{x}(\mathrm{x}-1) / \mathrm{KV}_{\mathrm{exc}}\right\}$

$\mathrm{dE}_{\mathrm{g}} / \mathrm{dx}$ is the variation of the energy gap with composition, for which we used the analytical expression $\mathrm{E}_{\mathrm{g}}(\mathrm{x})=\mathrm{E}_{\mathrm{g}, \mathrm{GaN}}+(\mathrm{c}-\mathrm{b}) \mathrm{x}+\mathrm{bx}^{2}$ with $\mathrm{c}=\mathrm{E}_{\mathrm{g}, \mathrm{AlN}}-\mathrm{E}_{\mathrm{g}, \mathrm{GaN}}$ and $\mathrm{b}=0.6 \mathrm{eV}$. For the band gaps we used $3.48 \mathrm{eV}(\mathrm{GaN})$ and $6.2 \mathrm{eV}(\mathrm{AlN}) . \mathrm{K}$ is the cation density. The factor 2.36 accounts for Gaussian broadening. The exciton volume is calculated from the Bohr radius for which we took $28 \AA$ in GaN and $19 \AA$ in AlN.

In fig. 6 we compare experiment and calculation. The maximum of broadening is reached for $70 \% \mathrm{Al}$ content (not shown in fig.6) which is related to the considerable increase of the electron effective mass towards AIN and the decrease of the dielectric constant. Due to the decreasing Bohr radius in $\mathrm{Al}_{\mathrm{x}} \mathrm{Ga}_{1-\mathrm{x}} \mathrm{N}$ the excitons will see much more alloy disorder.

In CL measurements (open diamonds) slightly different line widths are observed due to fact that the exciting spot size is smaller. In any case the experimental data are below the calculated. We have to have in mind, however, that we are dealing in the experiments with donor bound excitons and not with free excitons for which the Bohr radius concept is valid. For neutral acceptor bound excitons which were treated in the framework of the pseudo-donor model the influence of alloy broadening could be calculated. However, this model cannot been transfered to neutral donor bound excitons, since it requires the knowledge which particle of the exciton, electron or hole is bound first. Theoretical arguments suggest that the difference in linewidth of a bound exciton and a free exciton at low temperatures may not be significant.

Our experimental results confirm that alloy broadening in this statistical model accounts for the line width and clustering is negligible at least for the composition range investigated. It is also in line with the wavelength imaging by CL presented in fig. 4 .

From X-ray data it was already concluded that $\mathrm{Al}_{\mathrm{x}} \mathrm{Ga}_{1-\mathrm{x}} \mathrm{N}$ grew coherently on GaN resulting in a tensile stress in the $\mathrm{Al}_{\mathrm{x}} \mathrm{Ga}_{1-\mathrm{x}} \mathrm{N}$ layers. It should, however, be less than $0.5 \%$ since strained and 
unstrained $\mathrm{Al}_{\mathrm{x}} \mathrm{Ga}_{1-\mathrm{x}} \mathrm{N}$ layers have practically the same composition dependence of the band gap ${ }^{10}$. Our data support these conclusions. There is no evidence for a Stokes shift between absorption and emission and the bowing parameter is small. In $\mathrm{Al}_{\mathrm{x}} \mathrm{Ga}_{1-\mathrm{x}} \mathrm{N}$ for the alloy composition range $0<\mathrm{x}<0.22$ which is also the technological relevant one for the realisation of blue laserdiodes variations in recombination energies are less than $15 \mathrm{meV}$. For $\mathrm{x}=0.22$ this translates into composition variations of less than $2 \%$. The films showed free exciton emission at room temperature and with very good precision its position on alloy composition can be approximated with a linear dependence for $0<x<0.22$ to be $\mathrm{E}_{\mathrm{g}}(\mathrm{GaN})+2.15 \mathrm{x}(\mathrm{eV})$.

\section{SUMMARY}

In conclusion we reported on photoluminescence, calorimetric spectroscopy and cathodoluminescence of $\mathrm{Al}_{\mathrm{x}} \mathrm{Ga}_{1-\mathrm{x}} \mathrm{N}$ on $\mathrm{GaN}$ layers for alloy compositions $0<\mathrm{x}<0.22$. Small compositional inhomogeneities could be resolved, but will play a minor if not negligible role in carrier localisation. The luminescence linewidth increases in the alloy as expected for a random alloy. In contrast to InGaN/GaN pseudomorphic $\mathrm{Al}_{\mathrm{x}} \mathrm{Ga}_{1-\mathrm{x}} \mathrm{N} / \mathrm{GaN}$ single heterostructures with $\mathrm{x}<0.22$ show a small bowing parameter ${ }^{11}$. There is an overall agreement in line positions of free exciton absorption and emission. Additional compressive strain is added to GaN layers by growing AlGaN films on top.

\section{References}

${ }^{1}$ G. Steude, D.M. Hofmann, B.K. Meyer, H. Amano, and I. Akasaki, physica status solidi (b) 205, R7 (1998)

${ }^{2}$ G. Steude, D.M. Hofmann, B.K. Meyer, H. Amano, and I. Akasaki, physica status solidi (a), 165, R3 (1998)

${ }^{3}$ T. Takeuchi, H. Takeuchi, S. Soat, H. Sakai, H. Amano, and I. Akasaki, Jpn. J. Appl. Phys. 36, L177 (1997)

${ }^{4}$ L. Podlowski, A. Hoffmann, I. Broser, J. Cryst. Growth 117, 698 (1992).

${ }^{5}$ J. Christen, M. Grundmann, D. Bimberg, J. Vac. Sci. Technol. B9, 2358 (1991).

${ }^{6}$ P. Lautenschlager, M. Garriaga, S. Logothetidis, and M. Cardona, Phys. Rev. B 35, 9174 (1987).

${ }^{7}$ G. D. Cody, in Semiconductors and Semimetals, edited by J.I.Pankove, (Academic, New York, 1984), Vol. 21 B, pp 11-79.

${ }^{8}$ G. Steude, B.K. Meyer, A. Hoffmann, F. Bechstedt to be published

${ }^{9}$ E.F. Schubert, E.O. Göbel, Y. Horikoshi, K. Ploog and H.J. Queisser, Phys. Rev. B 30, 813 (1984)

${ }^{10}$ D.K. Wickenden, C.B. Bargeron, W.A. Byrden, J. Miragliova, and T.J. Kistenmacher, Appl. Phys. Lett. 65, 2024 (1994)

${ }^{11}$ M.D. McCluskey, C.G. Van de Walle, C.P. Master, L.T. Romero, and N.M. Johnson, Appl. Phys. Lett. 72, 2725 (1998) 